\title{
Photochemical grafting of methyl groups on a Si(111) surface using a Grignard reagent.
}

\section{AUTHOR(S):}

Herrera, Marvin Ustaris; Ichii, Takashi; Murase, Kuniaki; Sugimura, Hiroyuki

\section{CITATION:}

Herrera, Marvin Ustaris ...[et al]. Photochemical grafting of methyl groups on a Si(111) surface using a Grignard reagent. J Journal of colloid and interface science 2013, 411: 145151

\section{ISSUE DATE:}

2013-12-01

URL:

http://hdl.handle.net/2433/179382

\section{RIGHT:}

(c) 2013 Elsevier Inc.; この論文は出版社版でありません。引用の際には 出版社版をご確認ご利用ください。; This is not the published version. Please cite only the published version. 
Photochemical Grafting of Methyl Groups on a Si(111) Surface using a Grignard Reagent

Marvin Ustaris Herrera, Takashi Ichii, Kuniaki Murase, Hiroyuki Sugimura*

Department of Materials Science and Engineering, Kyoto University, Yoshida-honmachi, Sakyo-ku, Kyoto 606-8501, Japan

email address of the authors:

herrera.ustaris.87s@st.kyoto-u.ac.jp

ichii.takashi.2m@kyoto-u.ac.jp

murase.kuniaki.2n@kyoto-u.ac.jp

hiroyuki-sugimura@mtl.kyoto-u.ac.jp

*corresponding author:

email: hiroyuki-sugimura@mtl.kyoto-u.ac.jp

address: Department of Materials Science and Engineering, Kyoto University,

Yoshida-honmachi, Sakyo-ku, Kyoto 606-8501, Japan

telephone number: $+81-75-753-9131$

fax number: $+81-75-753-9130$ 


\begin{abstract}
The photochemical grafting of methyl groups onto an n-type $\operatorname{Si}(111)$ substrate was successfully achieved using a Grignard reagent. The preparation involved illuminating a hydrogen-terminated $\mathrm{Si}(111)$ that was immersed in a $\mathrm{CH}_{3} \mathrm{MgBr}-\mathrm{THF}$ solution. The success was attributed to the ability of the n-type hydrogenated substrate to produce holes on its surface when illuminated. The rate of grafting methyl groups onto the silicon surface was higher when a larger illumination intensity or when a substrate with lower dopant concentration was used. In addition, the methylated layer has an atomically flat structure, has a hydrophobic surface and has electron affinity that was lower than the bulk Si.
\end{abstract}

KEYWORDS. methyl-terminated $\mathrm{Si}$; photochemical grafting; Grignard reagent; self-assembled monolayer 


\section{Introduction}

Photochemical technique has been used to graft organic molecules onto the $\mathrm{Si}$ surface [1-7]. The use of electromagnetic radiation with photon energy that is greater than the band gap of $\mathrm{Si}$ (e.g., UV and visible light) is capable of producing photogenerated charges, which can be utilized to graft organic molecules. Crucial to the generation of the photoinduced charges is the existence of a depletion layer (with accompanying semiconductor barrier). It is in this layer, in which the illumination-induced electron-hole pairs split and depending on the direction of electric field within this layer, one kind of charges goes to the Si surface. With this, the nature of illumination-depletion layer interaction dictates the kind (whether positive or negative charges) and quantity of charges that can go to the Si surface to promote grafting. Thus, this paper will show how illumination and the nature of depletion layer could possibly affect the grafting process.

Photochemical technique was mostly been used for grafting molecules with double bonds at one of its end (e.g., 1-alkenes). Illumination could generate Si surface charges, which can trigger the breakage of one of these double bonds and give way to the formation of Si-C bond. However, the case for Grignard reagent is different. Grignard reagent needs to undergoes oxidative decomposition, in order for it to release the alkyl radical that is needed to promote grafting. Patterned from the model presented by Fellah, et. al. [8-9], grafting using Grignard reagent involves the following reaction:

$$
\begin{aligned}
& \mathrm{RMgX}+\mathrm{p}^{+} \rightarrow \mathrm{R} \cdot+\mathrm{MgX}^{+} \\
& \equiv \mathrm{Si}-\mathrm{H}+\mathrm{R} \cdot \rightarrow \equiv \mathrm{Si} \cdot+\mathrm{RH}
\end{aligned}
$$




$$
\equiv \mathrm{Si} \cdot+\mathrm{R} \cdot \rightarrow \equiv \mathrm{Si}-\mathrm{R}
$$

Equation 1 shows the oxidative decomposition of Grignard reagent into alkyl radical $(\mathrm{R} \cdot)$ and positively charged Magnesium Halide $\left(\mathrm{MgX}^{+}\right)$upon the introduction of positive charge. Meanwhile, equation 2 shows the formation of the silicon radical $(\mathrm{Si} \cdot)$ as the result of the interaction of the alkyl radical $(\mathrm{R} \cdot)$ with the hydrogen-terminated silicon ( $\mathrm{Si}-\mathrm{H})$. Lastly, equation 3 shows the grafting of the alkyl molecule $(\mathrm{Si}-\mathrm{R})$ as the result of the interaction between the silicon radical $(\mathrm{Si} \cdot)$ and the alkyl radical $(\mathrm{R} \cdot)$.

The oxidative decomposition of the Grignard reagent by the induction of positive charge (Equation 1) is a prerequisite for alkyl grafting to proceed. The oxidative decomposition of the Grignard reagent could be achieved either by means of the electron capture by the reduction of alkyl halide [8], by anodization [9], or by combined anodization and illumination [10]. Furthermore, we have shown that it can also be achieved by illumination alone without the use of an external voltage source [11].

Methyl groups on silicon surfaces have been subject of many studies [12-23]. Methyl groups have the ability to form stable $\mathrm{Si}-\mathrm{C}$ bonds with the silicon surface and are the smallest among the alkyl group. The combination of these two qualities creates a unique advantage among other organic groups that have been grafted onto silicon surfaces. Compared with small atoms, such as those of hydrogen and of halides, methyl groups can establish stronger and more stable bonds with the silicon surface. Likewise, compared with other alkyl group that can also establish strong and stable covalent bonds 
with the silicon surface, methyl groups are small enough to fit in between the $\mathrm{Si}(111)$ spacing, and thus are able to fully passivate the entire silicon surface with Si-C bonds. This small size allows them to fit in between large organic molecules that are grafted on the $\operatorname{Si}(111)$ surface and establish Si-C bonds on sites left vacant due to the geometric considerations. The establishment of $\mathrm{Si}-\mathrm{C}$ bonds on such unbounded sites can prevent oxidization that could otherwise be detrimental to the intended usage of the grafted molecules.

The attachment of organic molecules onto silicon surfaces [24-34] has been cited in different reports and several methods have been used to achieve such attachment. In alkyl groups grafting in particular, the chlorination-alkylation method [14-15, 19-24, 34], electrochemical method [9, 13, 16-18, 34], and photoanodic method [10] using Grignard reagent have been employed. However, a light-based technique would also be advantageous because it can be adaptable with photopatterning technique that would enable to make organic molecule-based spatial circuit patterns on the $\mathrm{Si}$ surface. Procedure-wise, unlike the chlorination-alkylation method, photochemical preparation is a simple method that uses hydrogen-terminated silicon directly, instead of an intermediate chlorine-terminated silicon. Similarly, unlike in electrochemical and photoelectrochemical methods, it does not require the placement of an ohmic contact between the silicon substrate and an external circuit that could induce damage to the sample.

The mechanism of the photochemical method deviates from those of the reduction of alkyl halide, electrochemical anodization, and the photoelectrochemical 
method. In the case of reduction of alkyl halide, the charge transport (directly involved in the oxidative decomposition of Grignard reagent) occurs within the solution, which is from the alkyl halide to Grignard reagent, rather than from the silicon substrate as in the case of photochemical preparation. In comparison with the electrochemical method, both of them involved holes coming from the sample's surface. However, the holes in the anodization method are induced by external voltage while those in the photochemical method are induced by illumination. In comparison with the photoelectrochemical method, both of them involve the presence of photogenerated holes. However, in the photoelectrochemical method, the electric field and the voltage between the electrodes can influence the charged particles (e.g. photogenerated holes, alkyl radicals) in the system to facilitate rapid alkylation. Such external electric field and voltage are absent in the photochemical method.

Analysis of the photochemical preparation technique is of scientific interest because it offers an opportunity to explore the mechanics of photogenerated charges vis-à-vis the grafting process. This will show how a semiconductor's band bending structure can affect the grafting process. Meanwhile, the authors believe that the detailed mechanics of how positive charges trigger alkylation is already extensively discussed in the literature [8-9], and thus the discussions of this report will focus on the aspect of the production of photogenerated charges. 
2. Experimental Procedure

\subsection{Wafer Cleaning and Hydrogen-termination}

The kinds of silicon wafer that were used (and their corresponding abbreviations) are as follows: (1) Phosphorus-doped Si(111) with resistivity of 1-10 $\Omega$ cm (n-low Si), (2) Phosphorus-doped Si(111) with resistivity of 0.001-0.004 $\Omega \mathrm{cm}$ (n-high Si), and (3) Boron-doped $\mathrm{Si}(111)$ with resistivity of 1-30 $\Omega \mathrm{cm}$ (p-low Si). The " $n$ " or " $p$ " in the abbreviation signifies the type of dopant in the silicon wafer. The term "n" was used for n-type dopant while the term "p" was used for p-type dopant. The terms "low" or "high" in the abbreviation signify the doping concentration of the sample. The term "low" was used for wafers with low dopant concentration or high resistivity (1-10 $\Omega \mathrm{cm}$ or 1-30 $\Omega \mathrm{cm}$ ) while the term "high" was used for wafers with high dopant concentration or low resistivity $(0.001-0.004 \Omega \mathrm{cm})$. All silicon wafers were single-sided and mirror-polished.

The cut silicon wafers were cleaned ultrasonically in ethanol and ultrapure water (UPW) for $20 \mathrm{~min}$ each. Then, the samples were photochemically cleaned using a vacuum ultraviolet (VUV) for $20 \mathrm{~min}$ to remove organic contaminants. The UV radiation, having a wavelength of $172 \mathrm{~nm}$ and intensity of $10 \mathrm{~mW} \cdot \mathrm{cm}^{-2}$, came from a xenon excimer lamp.

The hydrogen-termination process was performed by immersing the cleaned samples in 5\% $\mathrm{HF}$ solution at room temperature and $40 \% \mathrm{NH}_{4} \mathrm{~F}$ solution in a $80^{\circ} \mathrm{C}$ water bath. The length of time the cleaned wafers were immersed in the $\mathrm{HF}$ and $\mathrm{NH}_{4} \mathrm{~F}$ 
solutions depends on the doping concentration of the samples. For wafers with low dopant concentrations (n-low Si or p-low Si), the immersion time in HF solution was 5 min while the immersion time in $\mathrm{NH}_{4} \mathrm{~F}$ solution was $30 \mathrm{~s}$. For wafers with high dopant concentration (n-high $\mathrm{Si}$ ), the immersion time in HF solution was $30 \mathrm{~s}$ while the immersion time in $\mathrm{NH}_{4} \mathrm{~F}$ solution was 1 min. After the hydrogen-termination process, the samples were dried using a stream of nitrogen. The different kinds of hydrogen-terminated $\mathrm{Si}(111)$ that were fabricated (and their corresponding abbreviations are as follows: (1) hydrogen-terminated $\mathrm{Si}(111)$ using an n-low $\mathrm{Si}$ substrate (n-low Si-H), (2) hydrogen-terminated $\mathrm{Si}(111)$ using an n-high Si substrate (n-high Si-H) and (3) hydrogen-terminated Si(111) using a p-low Si substrate (p-low Si-H).

\subsection{Methyl-termination}

CAUTION: The methyl-termination preparations used a Grignard reagent. Extreme caution should be done in handling this chemical since it reacts vigorously with water, acids, alcohols and amines. In addition, the Grignard reagent also burns spontaneously without solvents. To ensure safety, the methyl grafting process, as well as the opening and storage of Grignard Reagent bottles were done inside a water vapor controlled chamber. In addition, all the materials that were used in the grafting process were carefully kept dry. Most importantly, the waste Grignard reagents were made to react with acetone and their by-product's $\mathrm{pH}$ was made to be near neutral before it was placed in the chemical waste container. This procedure was done to ensure the safe 
disposal of Grignard reagent.

The photochemical preparation was performed by illuminating visible light on a hydrogen-terminated $\mathrm{Si}(111)$ sample that was immersed in a Grignard Reagent-tetrahydrofuran solution $\left(\mathrm{CH}_{3} \mathrm{MgBr}-\mathrm{THF}\right)$ at room temperature. A quartz vessel with a rectangular-shaped bottom and a cylindrical upper body was used as an optical cell for the photochemical preparation process. One mol/1 (12 \%) $\mathrm{CH}_{3} \mathrm{MgBr}-\mathrm{THF}$, which served as the grafting electrolyte, was placed inside the vessel. The cell was covered with a silicone stopper having an inlet and outlet for nitrogen gas. The solution was bubbled with nitrogen gas prior to and during the grafting process. A reservoir bottle filled with THF was placed along the nitrogen line to compensate for the THF that would be lost because of its volatility. The visible light used originated from a xenon lamp (Asahi Spectra Co., Ltd., Max 1000). The light intensity emerging from the optical fiber could be controlled using a built-in device and its spectrum [4] is published elsewhere.

\subsection{Postcleaning Treatment}

After the photochemical preparation, the prepared samples were rinsed with THF and then cleaned ultrasonically using ethanol and UPW for $10 \mathrm{~min}$ each. The samples were then dried using a stream of nitrogen. Postcleaning treatment was done to remove residues of the photochemical preparation and other impurities.

\subsection{Analytical Tools}


X-ray photoelectron spectroscopy (XPS) was used to obtain the O 1s, C 1s and Si 2p spectra of the samples. An XPS system (Kratos Analytical Ltd., ESCA-3400) was used for this purpose. The X-ray source was $\mathrm{MgK} \alpha$ working at an emission current of $10 \mathrm{~mA}$ and an acceleration voltage of $10 \mathrm{kV}$. The scans were done with a step size of $0.1 \mathrm{eV}$ for $298.5 \mathrm{~ms}$ and repeated 10 times. The XPS quantitative analysis was done using the built-in software in the device.

The ATR-FTIR spectrum was taken in a sample chamber of a Digilab FTIR Spectrometer (Excalibur FTS 3000). A GATR ${ }^{\mathrm{TM}}$ single reflection horizontal ATR accessory, which employs a Ge ATR crystal and a $65^{\circ}$ angle of incidence, was used. The spectrum was recorded using Digilab Resolutions Pro 4.0 software package.

A water contact angle meter (Kyowa Interface Science Co., Ltd., DM 500) was used to measure the static water contact angle of the samples. In this method, water droplets with a volume of $1.8 \mu \mathrm{l}$ were used for the measurements.

An atomic force microscope (Asylum Research, MFP-3D-SA AFM) with an aluminum-backside coated Si probe (SII NanoTechnology Inc., SI-DF20) in AC mode was used to obtained the surface topographical images of the sample.

The ionization potential of the methylated surface was determined using a photoemission yield spectroscopy (PYS) system (Riken-Keiki Co., Ltd., Surface Analyzer AC-2). The sample was illuminated with $3 \mathrm{nW}$ ultraviolet light, scanned from 4.20 to $6.00 \mathrm{eV}$ in $0.05 \mathrm{eV}$ steps. 
3. Results and Discussion

\subsection{Photochemical grafting process}

Methyl-terminated $\mathrm{Si}(111)$ on an n-type substrate was successfully fabricated using a photochemical grafting technique. However, we were not successful in producing a methyl-terminated surface on a p-type substrate using the said photochemical preparation.

Figure 1 shows the Si $2 p$ spectra of the different samples. Figures 1a and 1c show the Si 2 p spectra of n-low $\mathrm{Si}-\mathrm{H}$ and p-low $\mathrm{Si}-\mathrm{H}$, respectively. Figure $1 \mathrm{~b}$ shows the Si $2 p$ spectrum of an $\mathrm{n}$-low $\mathrm{Si}-\mathrm{H}$ that underwent photochemical preparation while Figure 1d shows the p-type counterpart. The hydrogen-terminated samples underwent the same post-cleaning treatment as the photochemical treated samples. The two possible peaks of $\mathrm{Si} 2 \mathrm{p}$ spectrum are associated with the following silicon-based materials: (1) crystalline silicon (large peak at lower binding energy) and (2) silicon oxide (small peak at higher binding energy). Figure 1 also shows an inset that highlights the region associated with the presence of silicon oxide. For comparison purposes, the spectra of the samples were normalized to facilitate comparison of the spectra.

The use of n-low Si-H in the photochemical preparation (Figure 1b) yielded a low silicon oxide level compared with the use of p-low Si-H (Figure 1d). The level of silicon oxide was even lower than that of H-terminated samples (Figures 1a and 1c). (To note, the levels of silicon oxide of the two $\mathrm{Si}-\mathrm{H}$ samples are almost the same.) The low 
level of silicon oxide was attributed with the presence of grafted methyl molecules that inhibited the formation of oxide. In contrast, the high silicon oxide level of the p-type sample was attributed to the absence (or low amount) of Si-C bonds that should have protected the surface from oxidation. The high silicon oxide level was also exacerbated by the presence of oxide-inducing photogenerated electrons that were present during the photochemical preparation.

Figure 2 shows the $\mathrm{C} 1 \mathrm{~s}$ spectrum of the n-type sample that underwent photochemical process. The spectrum shows peak asymmetry towards the higher binding energy. This peak asymmetry could be due to contaminations that cling on the surface during the photochemical grafting process and post-cleaning treatment (e.g., THF and ethanol).

Figure 3 shows the ATR-FTIR of the sample with n-type substrate that underwent photochemical grafting process for 210 min under $600 \mathrm{~mW} \cdot \mathrm{cm}^{-2}$ illumination. The vertical axis is in terms of $\Delta \mathrm{I} / \mathrm{I}$. The term "I" is the ATR signal of a hydrogen-terminated silicon, which served as the reference. For presentation purposes, the ATR-FTIR is divided into three regions, they are: (1) $700-1800 \mathrm{~cm}^{-1}$ (Figure 3a), $1800-2700 \mathrm{~cm}^{-1}$ (Figure 3b), and 2700-3050 $\mathrm{cm}^{-1}$ (Figure 3b). The symbols “"|" (parallel) and " $\perp$ " (perpendicular) indicate the direction of the IR vibration modes with respect to the surface of Si. Table 1 shows the different peaks of the ATR-FTIR profile.

The ATR-FTIR profile (Figure 3a) of the methyl-terminated Si(111) shows a high peak at around $1255 \mathrm{~cm}^{-1}$. This peak is attributed with the $\mathrm{C}-\mathrm{H}$ symmetric bending/deformation mode $\left(\delta_{\mathrm{s}}\left(\mathrm{CH}_{3}\right)\right)$ or $\mathrm{Si}-\mathrm{CH}_{3}$ umbrella mode [12-13, 20,35], which is 
perpendicular to the Si surface. On the other hand, the peak due to the $\mathrm{CH}_{3}$ rocking mode $\left(\rho\left(\mathrm{CH}_{3}\right)\right)$ (which is located at $751 \mathrm{~cm}^{-1}$ ) is very weak. $\mathrm{CH}_{3}$ rocking mode is oriented parallel to the Si surface. The peaks at 2958 and $2908 \mathrm{~cm}^{-1}$ in Figure 3c are attributed with the $\mathrm{CH}_{3}$ asymmetric $\left(v_{\mathrm{a}}\left(\mathrm{CH}_{3}\right)\right)$ and symmetric $\left(\mathrm{v}_{\mathrm{s}}\left(\mathrm{CH}_{3}\right)\right)$ stretching, respectively. Meanwhile, peaks are also seen at 2928 and $2854 \mathrm{~cm}^{-1}$. These peaks could be attributed with the $\mathrm{CH}_{2}$ asymmetric $\left(\mathrm{v}_{\mathrm{a}}\left(\mathrm{CH}_{2}\right)\right)$ and symmetric $\left(\mathrm{v}_{\mathrm{s}}\left(\mathrm{CH}_{2}\right)\right)$ stretching modes, respectively. These peaks could come from organic impurities that cling on the surface during the methylation process and the post-cleaning treatment. These impurities may have also caused the asymmetry in the $\mathrm{C} 1 \mathrm{~s}$ spectra of a methyl-terminated $\mathrm{Si}$. To note, $\mathrm{CH}_{2}$ infrared peaks are also seen in other methyl-terminated surfaces $[12,20]$. The negative peak at $2082 \mathrm{~cm}^{-1}$ in the ATR-FTIR profile (Figure $3 b)$ shows the stretching mode of $\mathrm{Si}-\mathrm{H}$ bonds $(\mathrm{v}(\mathrm{Si}-\mathrm{H}))$. This peak is initially presence in the hydrogen-terminated Si but diminished during methylation, thus exemplified as negative peak.

Equation 1 shows the importance of the positive charge in triggering the oxidative decomposition of Grignard reagent and promoting methylation. In this respect, the nature of the electronic structure of a hydrogen-terminated silicon, which is the precursor of methyl-terminated silicon, dictates the mechanics of how charges can be made available on the silicon surface and promotes methylation. Figure 4 shows the band diagram of H-terminated Silicon (a) with an n-type substrate (n Si-H) and (b) with a p-type substrate (p Si-H). Assume that both the H-terminated silicon materials are not connected to an external potential and are placed at the dark. The conduction $\left(\mathrm{E}_{\mathrm{C}}\right)$ and 
valence $\left(E_{V}\right)$ bands of the hydrogen-terminated surfaces bend and create a depletion layer with charge lumping in this layer. The energy bands of the $\mathrm{n} \mathrm{Si-H}$ bend downward (Figure 4a) while those of $\mathrm{p} \mathrm{Si-H} \mathrm{bend} \mathrm{upward} \mathrm{(Figure} 4 \mathrm{~b}$ ). The formation of the depletion region is accompanied by the formation of a semiconductor barrier that can prevent charge transfer from the bulk silicon out to its surface. This offers a possible explanation of why we were not successful in producing a methylated surface using p-type substrate in the dark even if the sample's majority carriers were holes. The presence of the semiconductor barrier makes it difficult for the majority carrier (holes) in the bulk to go to its surface.

There are two possible ways for positive charges to be released onto the semiconductor's surface; they are (1) by placing an external voltage on the sample's substrate and (2) by illumination of the sample's surface.

Positive charges can be made available on the sample's surface by connecting either the n-type or the p-type substrate with sufficient external positive bias. A positively biased n-type substrate can create an inversion layer that makes its surface rich with its minority carrier, which in this case are positive charges. Meanwhile, a positively biased p-type substrate can create an accumulation layer that makes its surface rich with its majority carrier, which in this case are also positive charges. It is important to note that the placement of an external voltage on an n-type [16-17,34] or a p-type $[9,13]$ substrate has been shown to produce methyl-terminated silicon. Likewise, the presence of an anodic current on both the $\mathrm{n} \mathrm{H}-\mathrm{Si}$ and the $\mathrm{p} \mathrm{H}-\mathrm{Si}$ electrodes in a $\mathrm{C}_{18} \mathrm{H}_{37} \mathrm{MgCl} / \mathrm{THF}$ solution in the dark [10] indicates the possibility of the oxidative 
decomposition of the Grignard reagent with anodization.

Illumination can induce the formation of positive charges on the sample's surface. The success of the photoelectrochemical method [10] shows that alkylation can be employed with the aid of both illumination and anodization. However, our success in producing methyl-terminated Si using the photochemical method on n-type substrate shows that even with photogenerated holes only (without the aid of external potential), the oxidative decomposition of Grignard reagent is also possible. However, the case would be different for the p-type sample in which the negative charges accumulate on the surface and promote oxidation.

\subsection{Rate of the Methyl Grafting}

Figure 5 shows a graph of the sample's oxygen atomic percent with grafting time. The decrease in the atomic percent of oxygen with grafting time indicates that the amount of surface covered with methyl group increases with time. Note that the " $0 \mathrm{hr}$ sample" is the n-low Si-H described in Figure 1b. The rate at which the atomic percent of oxygen decreases is slower at higher grafting time (120 to $240 \mathrm{~min}$ ) compared with the lower grafting time ( 0 to $120 \mathrm{~min}$ ). This is expected since the grafting rate must be dependent on the available grafting sites that decrease with time.

Crucial to the rate of the methyl grafting is the rate at which positive charges accumulate on the sample's surface. When illumination is absorbed at the sample's surface, this will induce electron-hole pair generation. The rate of such electron-hole pair or optical generation [36-37] decreases exponentially with the depth from the 
illuminated surface. In the depletion region (Figure 4), the electric field causes these charge pairs to separate, thus generating a charge drift within this region. (For simplicity, let us neglect the effect of possible electron-hole pair generation that may arise beyond the depletion region). For an n-type substrate, the separated holes will move onto the sample's surface and gain the ability to trigger methylation. The rate of hole accumulation at the surface is influenced (1) by the rate of the electron-hole pair generation and (2) by the width of the region (depletion region) in which the splitting of electron-hole pairs occurs.

The rate of the electron-hole pair generation or optical generation depends on the effective photon flux hitting the sample's surface which in turn is affected by (1) the quantum efficiency of the sample, (2) the transmittance of the grafting medium and (3) the illumination intensity used during grafting. The effect of the electron-hole pair generation rate on the methyl grafting process was investigated by varying the photochemical preparation's illumination intensity. Other factors that may affect the electron-hole pair generation were kept or assumed to be constant such as the nature of the hydrogen-terminated silicon on the n-type substrate (which affects the quantum efficiency of the sample) and the concentration of Grignard Reagent-THF solution (which affect the transmittance of the grafting medium). Table 2 shows the XPS quantitative analytical data of samples that were prepared for $2 \mathrm{hr}$ under different illumination intensities. The table shows that the oxygen atomic percent decreased with increasing illumination intensity. This indicates that the amount of grafted methyl molecules increased with the illumination intensity. A higher illumination intensity thus 
increases the rate of positive charge accumulation at the sample's surface, in turn triggering faster methylation. To note, it would be difficult to describe the amount of grafted methyl group based from the obtained carbon atomic percents because sufficiently large signals from carbon-based impurities are superimposed on the $\mathrm{C} 1 \mathrm{~s}$ spectra as shown in Figure 2.

The depletion layer is a space charge region wherein the carrier density is different from that of the bulk. Since the charge density in this region deviates from that of the bulk, a non-zero electric field exist in this region even when the external bias is zero. It is in this layer that the electric field splits the electron-hole pairs and one of them will go to the surface. Samples with a thinner depletion layer have fewer regions where the splitting of electron-hole pairs will occur, thus translating to lower accumulation of positive charges on the surface and a slower methylation process. The width of the depletion layer can be influenced by the dopant concentration. In this respect, substrates with a higher dopant concentration (low resistivity) will have a thinner depletion layer. Thus, it would be expected that such samples would have a slower methylation process compared to samples with a low dopant concentration substrate. Table 3 shows that the methyl-terminated silicon prepared on a low-doped substrate had a lower oxygen atomic percent than that of a high-doped substrate. This implies that the rate of methyl grafting on low-doped substrates is faster than that on high-doped substrates. 


\subsection{The Methyl-terminated Surface}

The methyl-terminated surface produced using photochemical preparation has an atomically flat structure, a hydrophobic surface and has lower electron affinity compared to that of the bulk Si.

Figure 6 shows the band diagram of methyl-terminated silicon. The methyl-terminated $\mathrm{Si}(111)$ surface has estimated surface potential step $(\delta)$ of $-0.31 \mathrm{eV}$. The surface potential step is the contribution of the surface dipole due to the grafted structure to electron affinity. It is taken to be the difference between the electron affinity of the sample and the electron affinity of the bulk silicon $\left(\chi_{\text {bulk Si }}=4.05 \mathrm{eV}\right)[38]$. The value of electron affinity $(\chi=3.74 \mathrm{eV})$ was calculated by subtracting the band gap of silicon $\left(E_{g}=1.12 \mathrm{eV}\right)$ from the value of the sample's ionization potential $\left(\mathrm{I}_{\mathrm{p}}=4.86 \mathrm{eV}\right)$. The ionization potential of the sample was estimated as the energy threshold of the plot of the square root of yield as a function of photon energy hitting the sample's surface (Figure 7). The threshold energy is taken as the crossing point of the background (1.7 $\mathrm{cps}^{1 / 2}$ ) and the yield line (with gradient of $25.95 \mathrm{cps}^{1 / 2} / \mathrm{eV}$ ).

The photochemical chemical preparation creates $\mathrm{Si}-\mathrm{C}$ bonds between the atoms of the silicon surface and the methyl group. These Si-C bonds, which are $\mathrm{sp}^{3}$-hybridized, should position the methyl group perpendicular to the $\mathrm{Si}(111)$ plane [20]. The van der Waal radius of a methyl group is also small enough to fit between the Si(111) spacing, thus avoiding overlapping of molecules. The methyl film on the Si(111) surface is thus atomically flat and can copy the structure of the hydrogen-terminated $\mathrm{Si}(111)$ surface. The AFM images of our methyl-terminated 
sample (Figure 8) show that even after the photochemical preparation, the stair-like structure of the hydrogen-terminated $\operatorname{Si}(111)$ [3] was preserved, and thus the methylated surface has atomically flat structure.

A highly dense methylated silicon surface is hydrophobic. Figure 9 shows the graph of water contact angle vs. grafting time. The water contact angle increased with grafting time up to $120 \mathrm{~min}$ when it reached $89.0 \pm 0.8^{\circ}$. The increase in the water contact angle at shorter grafting times was attributed to the increase in the coverage of methylation. However, when the sample was grafted for $240 \mathrm{~min}$, its value fell to $86.2 \pm$ $0.3^{\circ}$. Long time of photochemical grafting might have degrading effects to the hydrophobicity of the methylated surface. To note, the values of the water contact angle of the samples that were prepared for $60 \mathrm{~min}$ and longer are higher than the reported value of methyl-terminated silicon that was produced using chlorination-alkylation method, i.e. $(78 \pm 2)^{\circ}[19]$.

\section{Conclusion}

Methyl-terminated $\mathrm{Si}(111)$ was successfully created using a photochemical grafting technique on an n-type sample. The success in producing a methyl-terminated surface using a photochemical grafting technique shows the ability of photogenerated holes to trigger the oxidative decomposition of Grignard reagent similar to that of reduction of alkyl halide [8] and by anodization [9]. The dependence of the grafting process on photogenerated holes makes grafting highly influenced by illumination intensity and by the doping concentration of the substrate. In such, the rate of methyl 
grafting improved with the use of high illumination intensity and the use of silicon substrate with low dopant concentration. The methyl-terminated surface was hydrophobic and had an atomically flat structure. The grafting process also has created a surface that has lower electron affinity compared to the bulk Si. Since this technique is illumination-based, it has the potential to be used in photopatterning to create spatial methylated patterns on the silicon surface. Such creation of spatial patterns is important in molecular electronics.

ACKNOWLEDGMENTS. This research was supported by KAKENHI (Grant-in-Aid for Scientific Research) from the Ministry of Education, Culture, Sports, Science and Technology of Japan, and a STARC Joint Research Program. 


\section{References}

1. B.J. Eves, Q-Y. Sun, G.P. Lopinski, H. Zuilhof, J. Am. Chem. Soc. 126 (2004) 14318.

2. M. Herrera, T. Ichii, K. Murase, H. Sugimura, Chem. Lett. 41 (2012) 1188.

3. H. Sano, H. Maeda, S. Matsuoka, K.-H. Lee, K. Murase, H. Sugimura, Jpn. J. Appl. Phys. 47 (2008) 5659.

4. H. Sano, M. Zhao, D. Kasahara, K. Murase, T. Ichii, H. Sugimura, Journal of Colloid and Interface Science 361 (2011) 259.

5. M.P. Stewart, J.M. Buriak, J. Am. Chem. Soc. 123 (2001) 7821.

6. H. Sugimura, S. Mo, K. Yamashiro, T. Ichii, K. Murase, J. Phys. Chem. C 117 (2013) 2480 .

7. Q.-Y. Sun, L.C.P.M. de Smet, B. van Lagen, M. Giesbers, P.C. Thune, J. van Engelenburg, F.A. de Wolf, H. Zuilhof, E.J.R. Sudholter, J. Am. Chem. Soc. 127 (2005) 2514.

8. S. Fellah, R. Boukherroub, F. Ozanam, J.-N. Chazalviel, Langmuir 20 (2004) 6359.

9. S. Fellah, A. Teyssot, F. Ozanam, J.-N. Chazalviel, J. Vigneron, A. Etcheberry, Langmuir 18 (2002) 5851.

10. S. Takakusagi, T. Miyasaka, K. Uosaki, J. of Electroanalytical Chemistry 599 (2007) 344.

11. M. Herrera, T. Ichii, K. Murase, H. Sugimura, Chem. Lett. 41 (2012) 902. 
12. S. R. Amy, D.J. Michalak, Y.J. Chabal, L. Wielunski, P.T. Hurley, N.S. Lewis, J. Phys. Chem. C 111 (2007) 13053.

13. A. Fidelis, F. Ozanam, J.-N. Chazalviel, Surface Science 444 (2000) L7.

14. R. Hunger, R. Fritsche, B. Jaeckel, W. Jaegermann, L.J. Webb, N.S. Lewis, Phys. Rev. B 72 (2005) 045317.

15. R. Hunger, R. Fritsche, B. Jaeckel, L.J. Webb, W. Jaegermann, N.S. Lewis, Surface Science 601 (2007) 2896.

16. T. Miyadera, A. Koma, T. Shimada, Surface Science 526 (2003) 177.

17. R. Okada, T. Miyadera, T. Shimada, A. Koma, K. Ueno, K. Saiki. Surface Science $552(2004) 46$.

18. S. S. S. Vegunta, J. N. Ngunjiri, J.C. Flake, Langmuir 25 (2009) 12750.

19. I. Waluyo, H. Ogasawara, M. Kawai, A. Nilsson, T. Yamada, J. Phys. Chem. C 114 (2010) 19004.

20. L. J. Webb, S. Rivillon, D.J. Michalak, Y.J. Chabal, N.S. Lewis, J. Phys. Chem. B $110(2006) 7349$.

21. T. Yamada, M. Kawai, A. Wawro, S. Suto, A. Kasuya, A. J. of Chem. Phys. 121 (2004) 10660.

22. H. Yu, L.J. Webb, R.S. Ries, S.D. Solares, W.A. Goddard, J.R. Heath, N.S. Lewis, J. Phys. Chem. B 109 (2005) 671.

23. H. Yu, L.J. Webb, J.R. Heath, N.S. Lewis, Appl. Phys. Lett. 88 (2006) 252111.

24. A. Bansal, X. Li, S.I. Yi, W.H. Weinberg, N.S. Lewis, J. Phys. Chem. B 105 (2001) 10266.

25. R. Boukherroub, S. Morin, F. Bensebaa, D.D.M. Wayner, Langmuir 15 (1999) 3831.

26. R. Boukherroub, Current Opinion in Solid State and Materials Science 9 (2005) 66. 
27. J.M. Buriak, Chemical Reviews 102 (2002) 1271.

28. R.L. Cicero, M.R. Linford, C.E.D. Chidsey, Langmuir 16 (2000) 5688.

29. M. R. Linford, C.E.D. Chidsey, J. Am. Chem. Soc. 115 (1993) 12631.

30. M.R. Linford, P. Fenter, P.M. Eisenberger, C.E.D. Chidsey, J. Am. Chem. Soc. $117(1995) 3145$.

31. A. B. Sieval, A.L. Demirel, J.W.M. Nissink, M.R. Linford, J.H. van der Maas, W.H. de Jeu, H. Zuilhof, E.J.R. Sudholter, Langmuir 14 (1998) 1759.

32. A.B. Sieval, R. Opitz, H.P.A. Maas, M.G. Schoeman, G. Meijer, F.J. Vergeldt, H. Zuilhof, E.J.R. Sudholter, Langmuir 16 (2000) 10359.

33. L. Touahir, P. Allongue, D. Aureau, R. Boukherroub, J.-N. Chazalviel, E. Galopin, A.C. Gouget-Laemmel, C.H. de Villeneuve, A. Moraillon, J. Niedziolka-Jonsson, F. Ozanam, J.S. Andresa, S. Sam, I. Solomon, S. Szunerits, Bioelectrochemistry 80 (2010) 17.

34. L.J. Webb, N.S. Lewis, J. Phys. Chem. B. 107 (2003) 5404.

35. G.A. Ferguson, K. Raghavachan, J. Chem. Phys. 125 (2006) 154708.

36. L. Kronik, Y. Shapira, Surface Science Reports 37 (1999) 1.

37. S.M. Sze, K.K. Ng, Physics of Semiconductor Devices, 3rd ed. John Wiley \& Sons: New Jersey, (2007); p 674.

38. T. He, H. Ding, N. Peor, M. Lu, D.A. Corley, B. Chen, Y. Ofir, Y. Gao, S. Yitzchaik, J.M. Tour, J. Am. Chem. Soc. 130 (2008) 1699. 


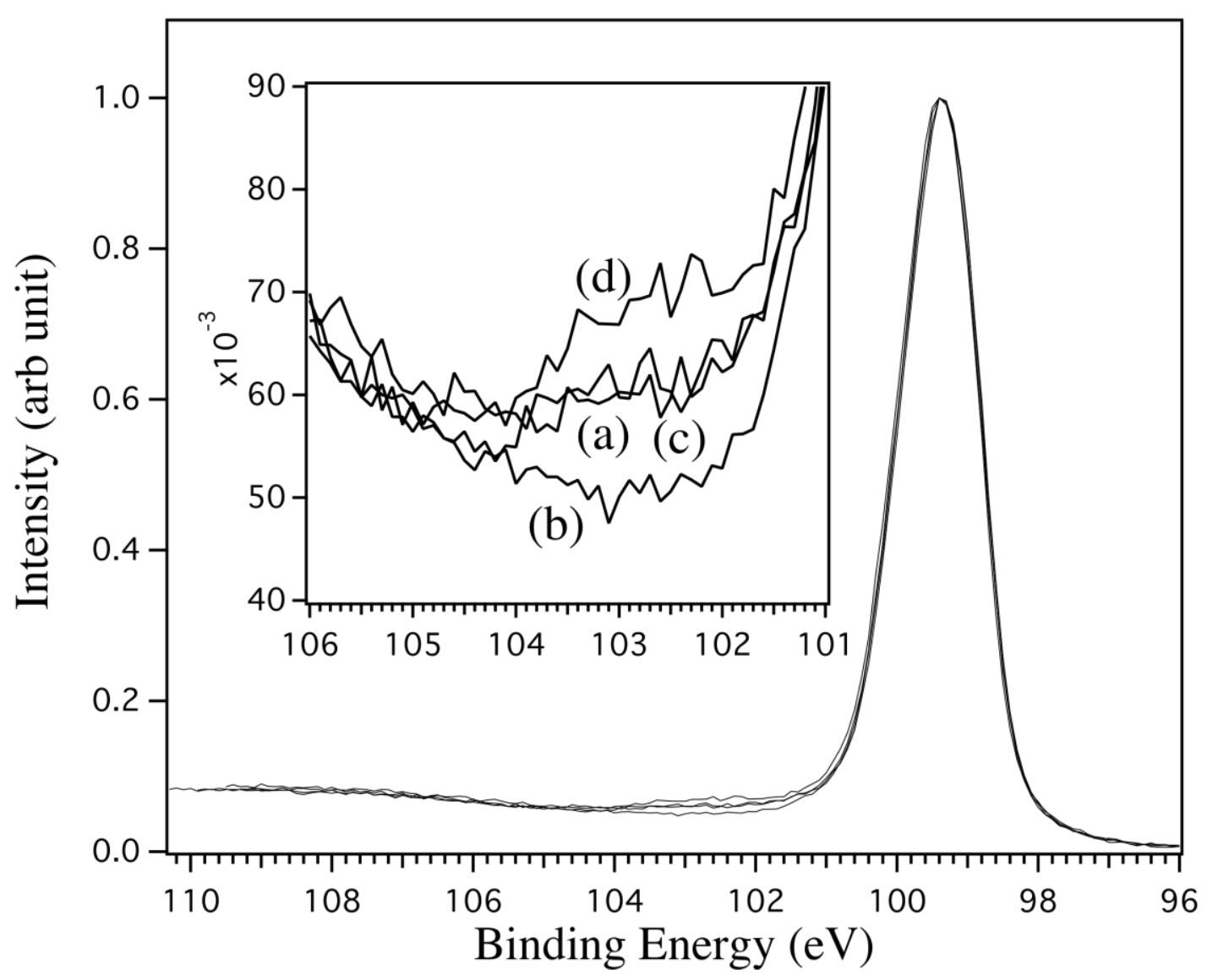

Figure 1 . Si $2 p$ spectra of the following samples: (a) hydrogen-terminated $\mathrm{Si}(111)$ with n-type substrate (n-low Si), (b) hydrogen-terminated $\mathrm{Si}(111)$ with n-type substrate (n-low Si) that was photochemically treated using $35 \mathrm{~mW} \cdot \mathrm{cm}^{-2}$ illumination, (c) hydrogen-terminated $\mathrm{Si}(111)$ with p-type substrate (p-low $\mathrm{Si}$ ), and (d) hydrogen-terminated $\mathrm{Si}(111)$ with p-type substrate (p-low $\mathrm{Si}$ ) that was photochemically treated using $35 \mathrm{~mW}-\mathrm{cm}^{-2}$ illumination. The inset shows the portion of Si $2 \mathrm{p}$ spectra associated with the silicon oxide. 


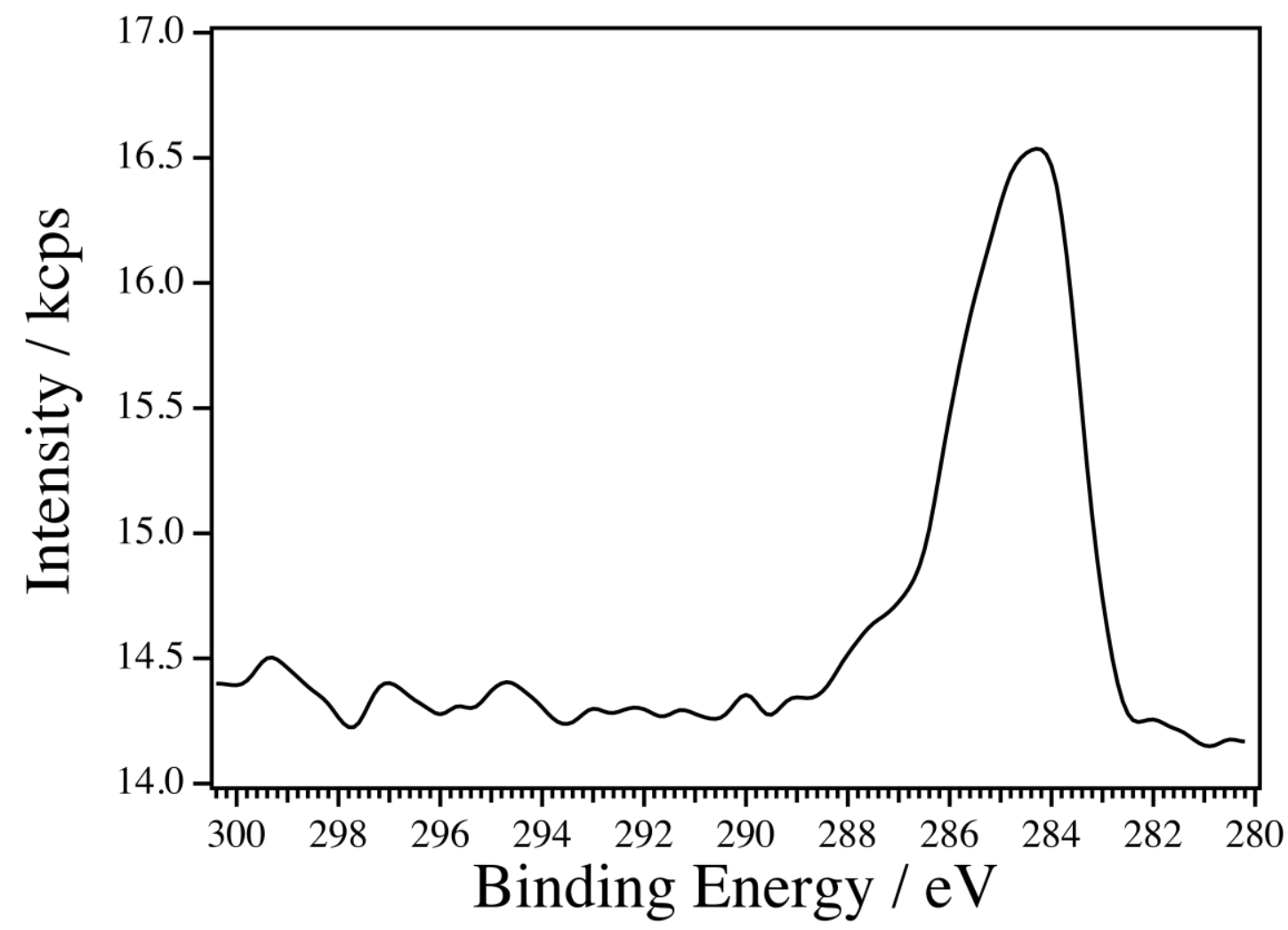

Figure 2. C 1s spectra of the methyl-terminated Si(111) sample 

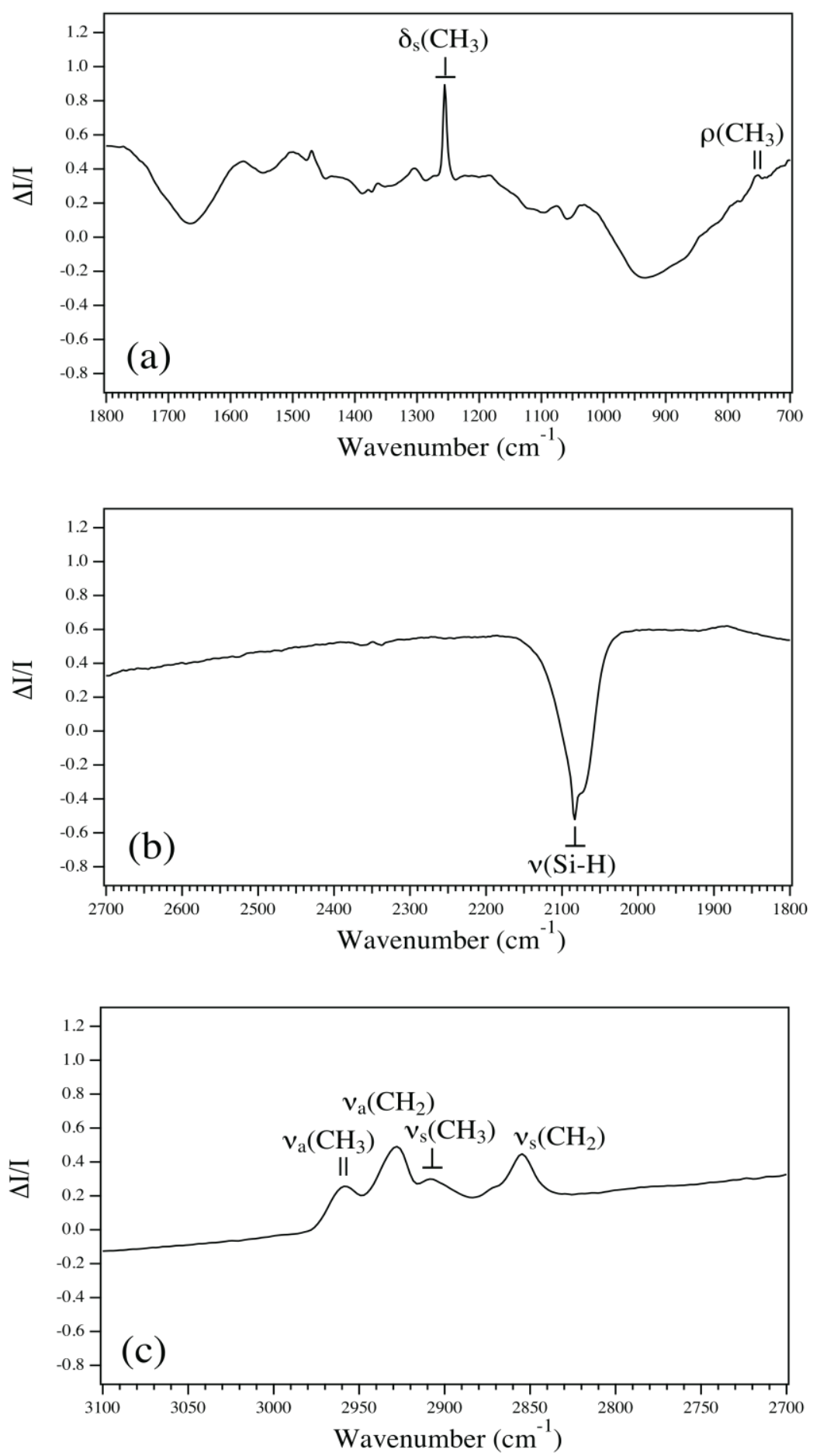

Figure 3. ATR-FTIR of a methyl-terminated $\mathrm{Si}(111)$ that was prepared using photochemical grafting technique 


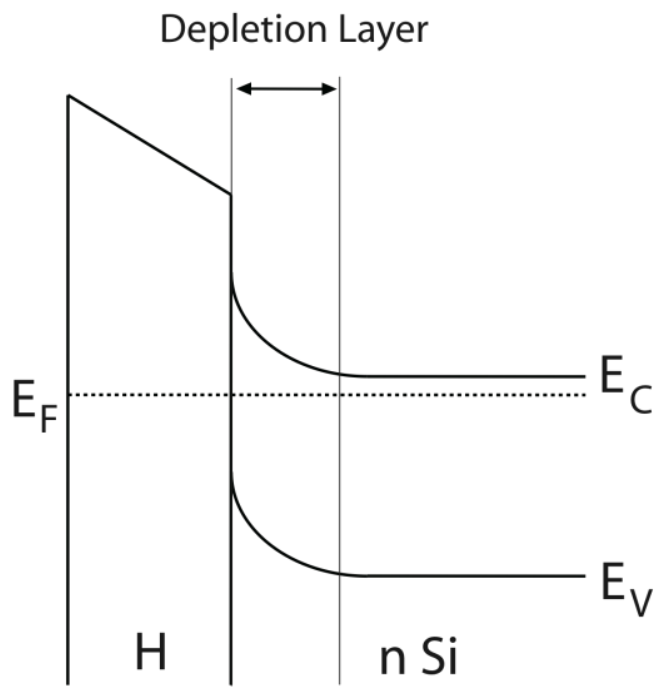

(a)

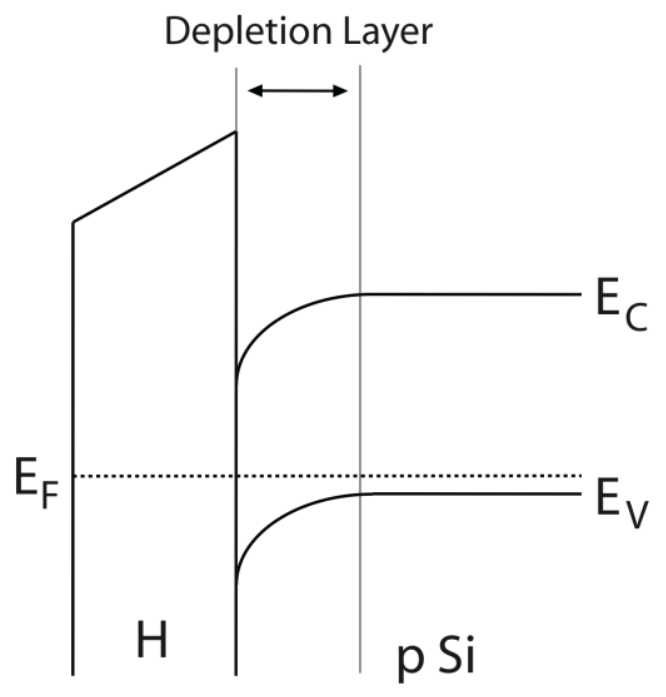

(b)

Figure 4. Band diagram of hydrogen-terminated Silicon with (a) n-type substrate (n $\mathrm{Si}-\mathrm{H})$ and (b) p-type substrate (p Si-H) in the dark that is not connected to an external voltage source. $\mathrm{E}_{\mathrm{c}}, \mathrm{E}_{\mathrm{v}}$ and $\mathrm{E}_{\mathrm{F}}$ denote conduction band, valence band and Fermi energies, respectively while " $\mathrm{n} \mathrm{Si"} \mathrm{and} \mathrm{"p} \mathrm{Si"} \mathrm{denote} \mathrm{n-type} \mathrm{and} \mathrm{p-type} \mathrm{silicon}$ substrates, respectively. 


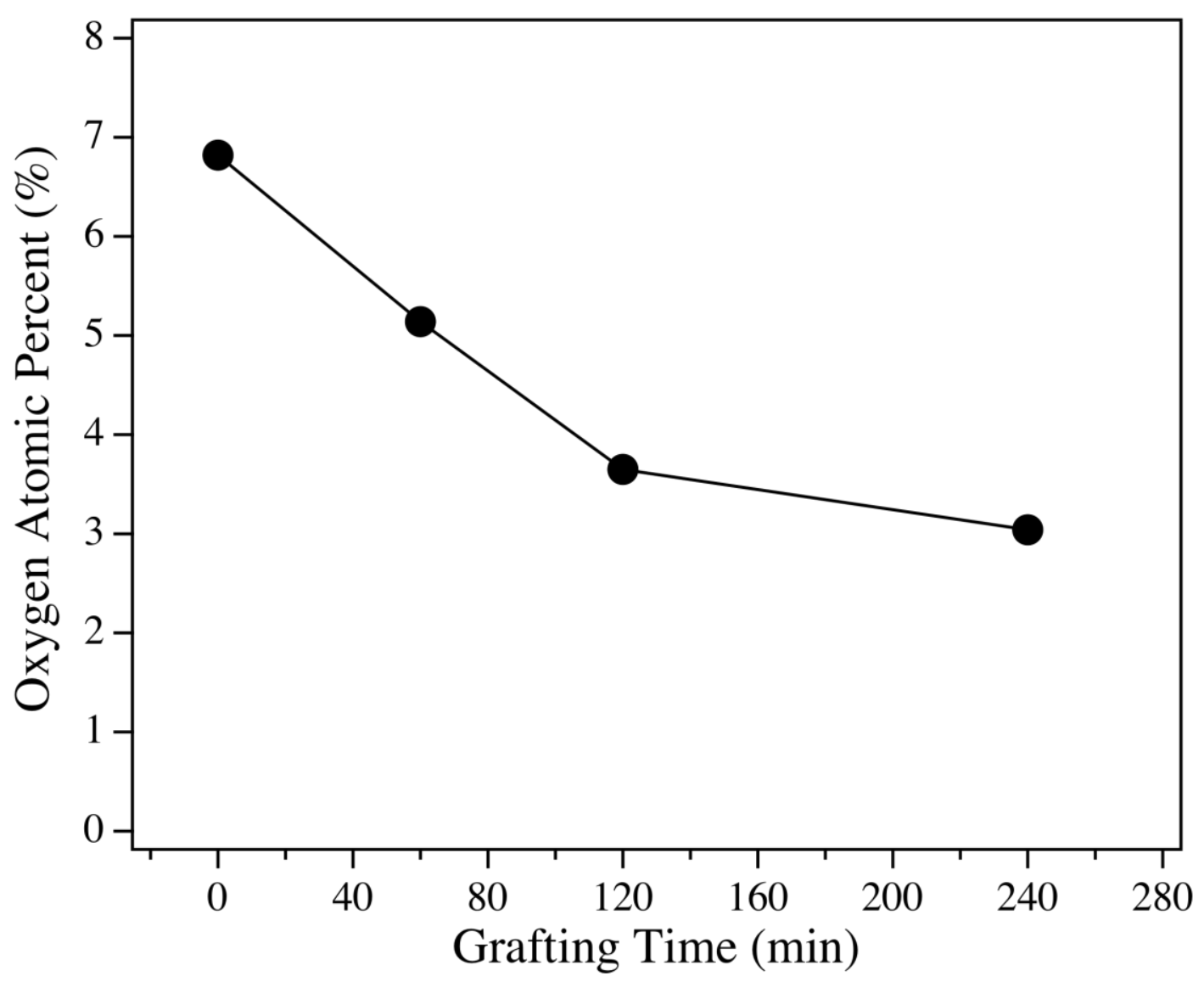

Figure 5. Grafting time vs. oxygen atomic percent of hydrogen-terminated $\mathrm{Si}(111)$ with n-type substrate with resistivity of $1-10 \Omega \mathrm{cm}$ that underwent photochemical preparation using $200 \mathrm{~mW} \cdot \mathrm{cm}^{-2}$ illumination. The oxygen atomic percent is based on the analysis of O 1s, C1s and Si 2p spectra. 


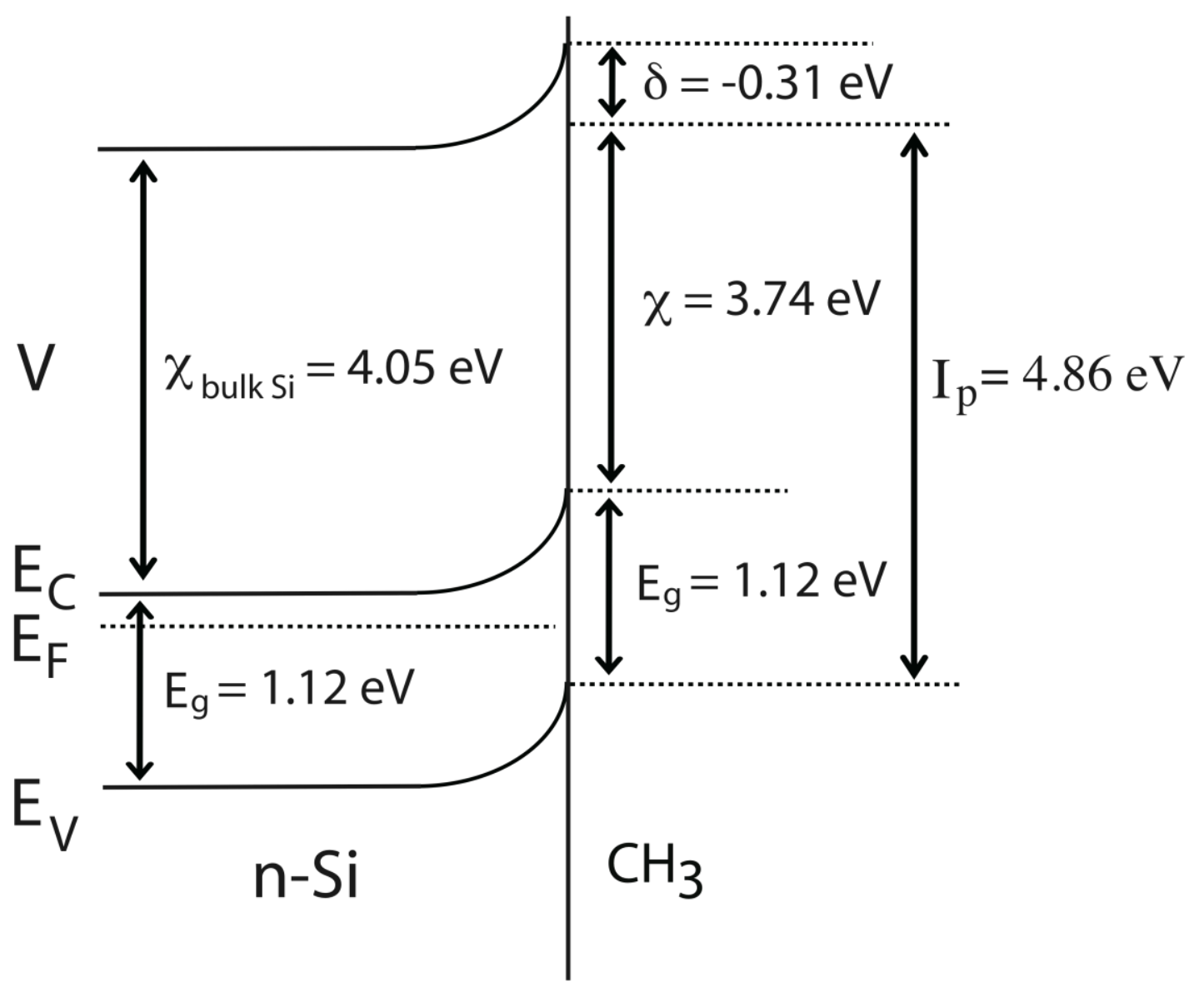

Figure 6. Band diagram of methyl-terminated $\mathrm{Si}(111)$ on n-type substrate. $\mathrm{V}, \mathrm{E}_{\mathrm{c}}, \mathrm{E}_{\mathrm{v}}$ and $\mathrm{E}_{\mathrm{F}}$ denote vacuum level, conduction band, valence band and Fermi energies, respectively. The terms $\chi$ bulk $S_{i}$ and $\mathrm{E}_{\mathrm{g}}$ are electron affinity and band gap energy of the bulk silicon, while the terms $\mathrm{I}_{\mathrm{p}}, \delta$ and $\chi$ are the ionization potential, surface potential step and electron affinity of the grafted surface, respectively. 


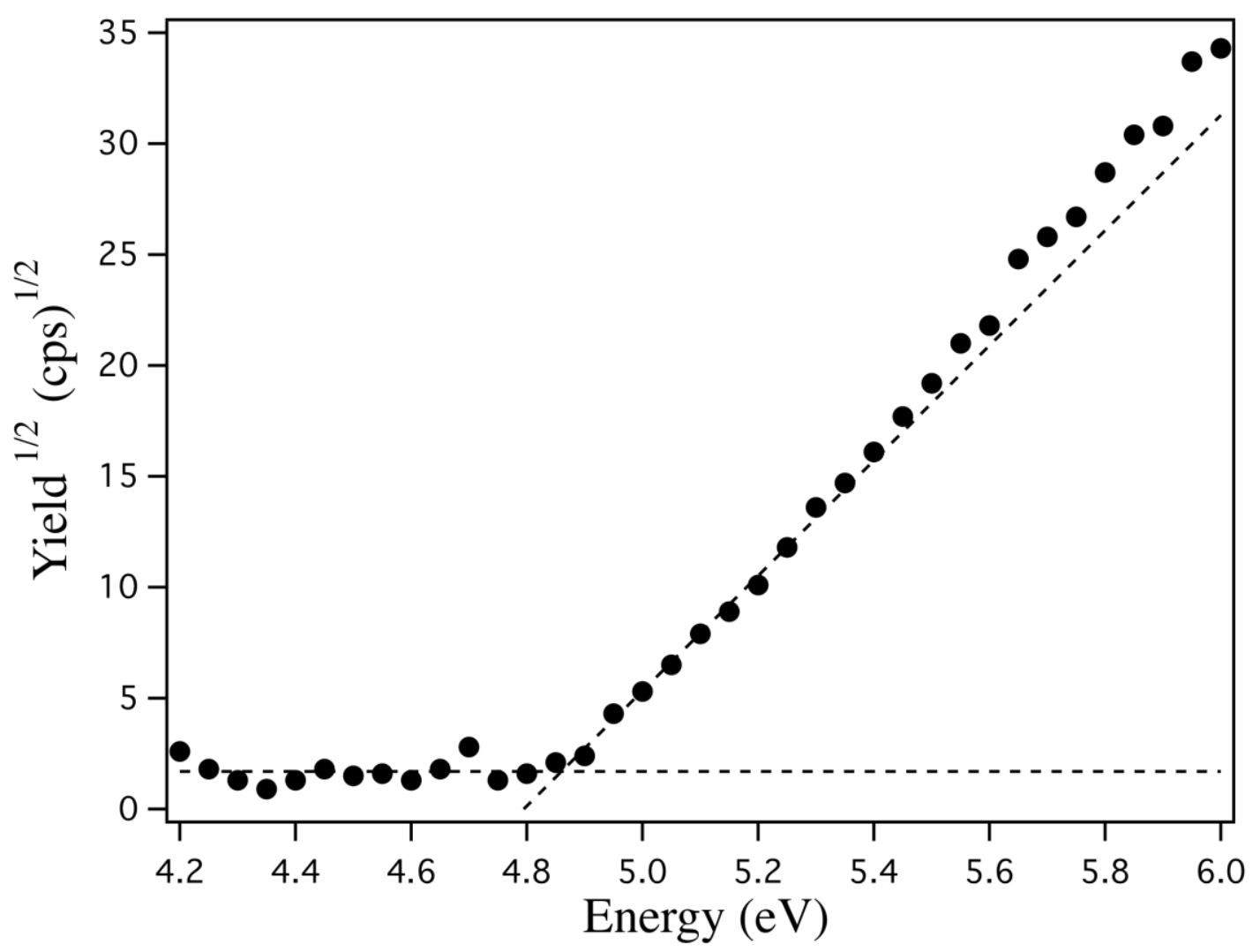

Figure 7. Graph of square root of photoemission yield vs. photon energy of a methyl-terminated $\mathrm{Si}(111)$ sample 


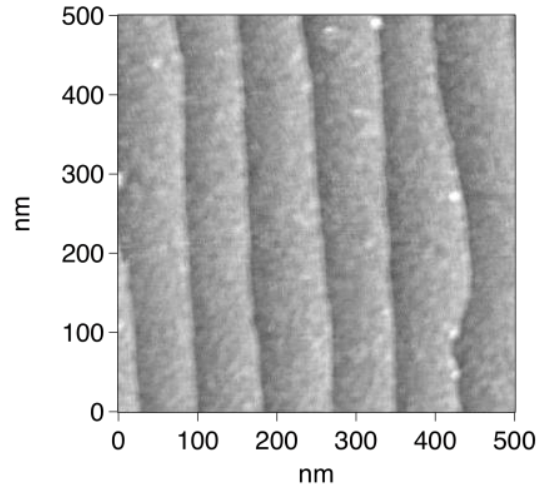

(a)

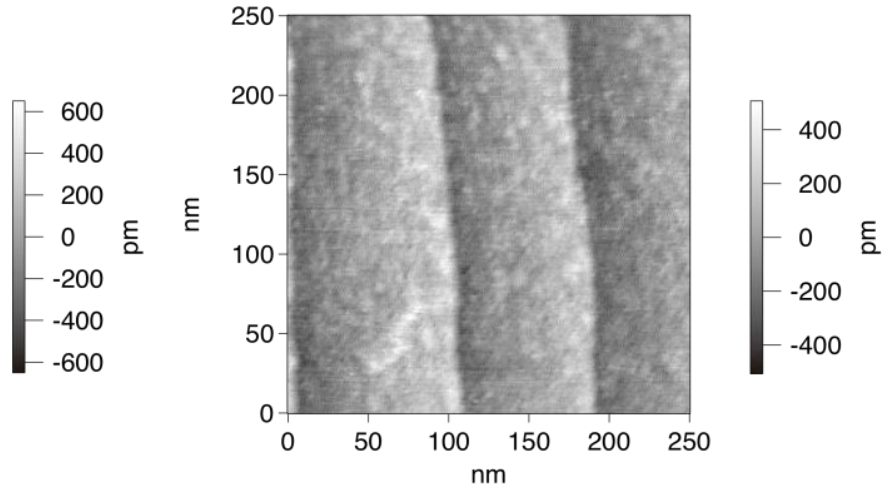

(b)

Figure 8. AFM topographical images of methyl-terminated $\operatorname{Si}(111)$ at (a) (500 x 500) $\mathrm{nm}$ and (b) (250 x 250) nm scan sizes 


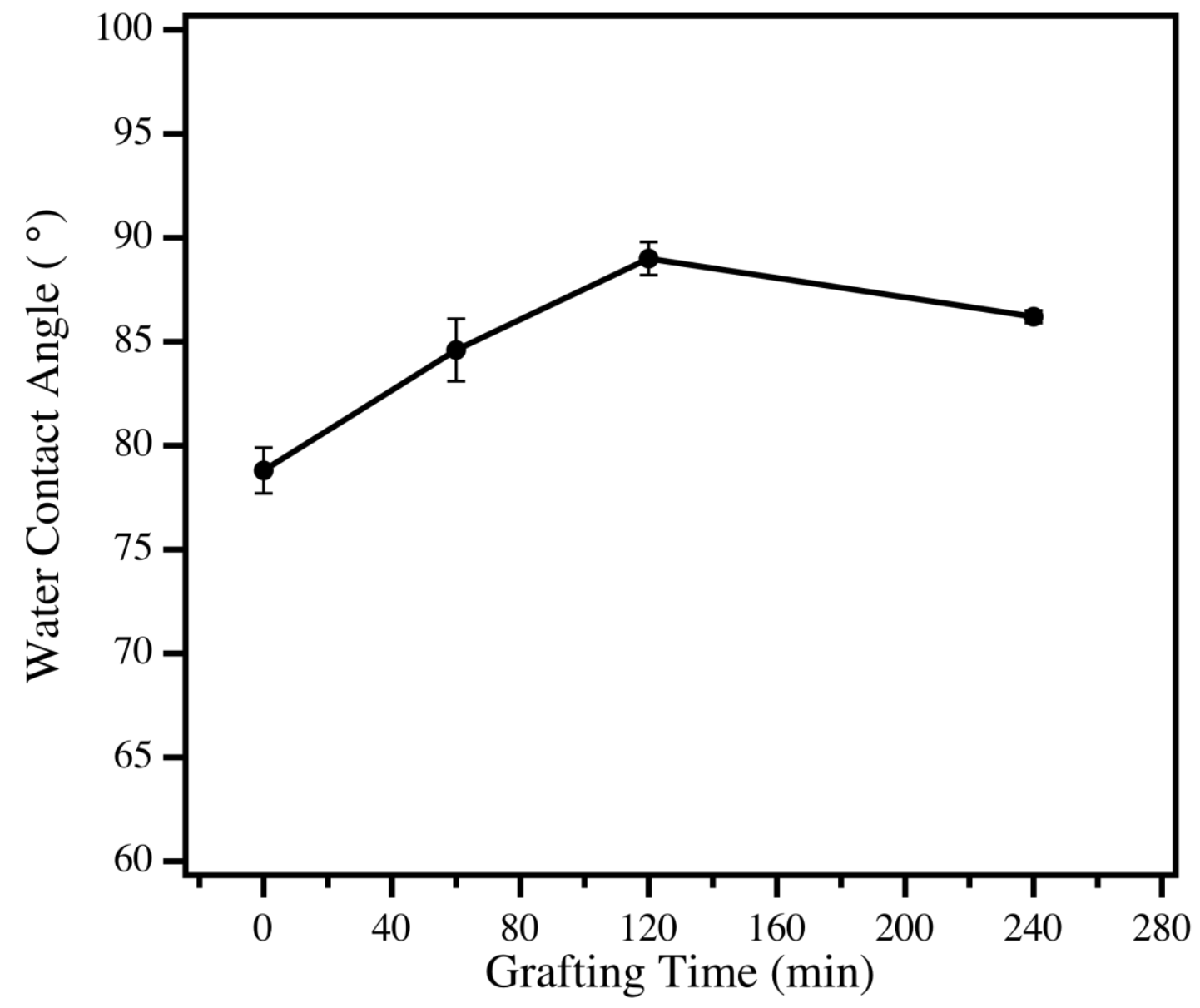

Figure 9. Grafting time vs. water contact angle of hydrogen-terminated $\mathrm{Si}(111)$ with n-type substrate having resistivity of 1-10 $\Omega \mathrm{cm}$ that underwent photochemical preparation using $200 \mathrm{~mW} \cdot \mathrm{cm}^{-2}$ illuminations. 
Table 1. ATR-FTIR spectra peaks of the methyl-terminated Si(111)

\begin{tabular}{|c|c|c|c|}
\hline Symbol & Mode & Wavenumber & $\|$ or $\perp$ to the $\mathrm{Si}$ \\
& & $\left(\mathrm{cm}^{-1}\right)$ & surface \\
\hline$v_{\mathrm{a}}\left(\mathrm{CH}_{3}\right)$ & Asymmetric stretching $\left(\mathrm{CH}_{3}\right)$ & 2958 & \\
\hline$v_{\mathrm{a}}\left(\mathrm{CH}_{2}\right)$ & Asymmetric stretching $\left(\mathrm{CH}_{2}\right)$ & 2928 & $\perp$ \\
\hline$v_{\mathrm{s}}\left(\mathrm{CH}_{3}\right)$ & Symmetric stretching $\left(\mathrm{CH}_{3}\right)$ & 2908 & $\perp$ \\
\hline$v_{\mathrm{s}}\left(\mathrm{CH}_{2}\right)$ & Symmetric stretching $\left(\mathrm{CH}_{2}\right)$ & 2854 & $\perp$ \\
\hline$v_{(\mathrm{Si}-\mathrm{H})}$ & Stretching $(\mathrm{Si}-\mathrm{H})$ & 2082 & $\|$ \\
\hline$\delta_{\mathrm{s}}\left(\mathrm{CH}_{3}\right)$ & Symmetric bending $\left(\mathrm{CH}_{3}\right)$ & 1255 & $\perp$ \\
\hline$\rho\left(\mathrm{CH}_{3}\right)$ & Rocking $\left(\mathrm{CH}_{3}\right)$ & 751 & $\perp$ \\
\hline
\end{tabular}


Table 2. XPS quantitative analytical data of hydrogen-terminated $\operatorname{Si}(111)$ with n-type substrate having resistivity of 1-10 $\Omega \mathrm{cm}$ that underwent photochemical preparation for $120 \mathrm{~min}$.

\begin{tabular}{|c|c|c|c|}
\hline $\begin{array}{c}\text { Illumination } \\
\text { Intensity } \\
\left(\mathrm{mW} \cdot \mathrm{cm}^{-2}\right)\end{array}$ & $\begin{array}{c}\mathrm{O} \\
(\text { at. \%) }\end{array}$ & $\begin{array}{c}\mathrm{C} \\
(\text { at. } \%)\end{array}$ & $\begin{array}{c}\mathrm{Si} \\
(\text { at. } \%)\end{array}$ \\
\hline 35 & 5.28 & 4.97 & 89.75 \\
\hline 70 & 5.09 & 5.24 & 89.67 \\
\hline 145 & 5.08 & 6.85 & 88.07 \\
\hline 200 & 3.65 & 6.61 & 89.74 \\
\hline 400 & 3.52 & 5.52 & 90.96 \\
\hline 600 & 2.46 & 7.65 & 89.89 \\
\hline
\end{tabular}


Table 3. XPS quantitative analytical data of hydrogen-terminated $\operatorname{Si}(111)$ with n-type substrate that underwent photochemical preparation for $60 \min$ using $200 \mathrm{~mW} \cdot \mathrm{cm}^{-2}$ illumination.

\begin{tabular}{|c|c|c|c|c|}
\hline Doping level & $\begin{array}{c}\text { Resistivity } \\
(\Omega \mathrm{cm})\end{array}$ & $\begin{array}{c}\mathrm{O} \\
(\text { at. \%) }\end{array}$ & $\begin{array}{c}\mathrm{C} \\
(\text { at. \%) }\end{array}$ & $\begin{array}{c}\mathrm{Si} \\
(\text { at. \%) }\end{array}$ \\
\hline n-low Si & $1-10$ & 5.14 & 7.18 & 87.69 \\
\hline n-high Si & $0.001-0.004$ & 6.98 & 11.15 & 81.87 \\
\hline
\end{tabular}


